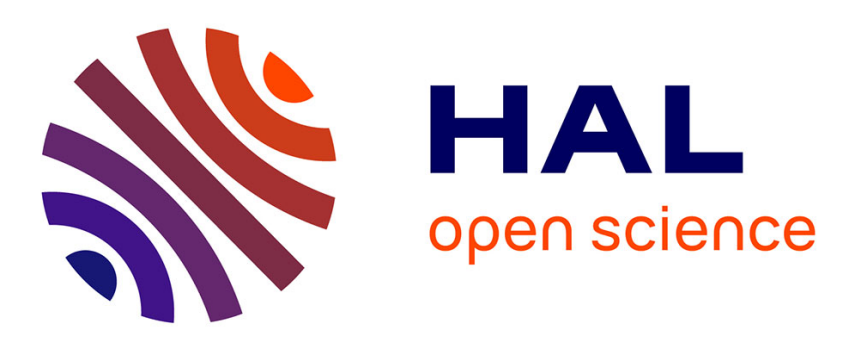

\title{
New insights into the tetrameric family of the Fur metalloregulators.
}

\author{
Serge Nader, Julien Pérard, Philippe Carpentier, L. Arnaud, Serge Crouzy, \\ Isabelle Michaud-Soret
}

\section{- To cite this version:}

Serge Nader, Julien Pérard, Philippe Carpentier, L. Arnaud, Serge Crouzy, et al.. New insights into the tetrameric family of the Fur metalloregulators.. BioMetals, 2019, 32 (3), pp.501-519. 10.1007/s10534019-00201-8 . hal-02166207

\section{HAL Id: hal-02166207 https://hal.science/hal-02166207}

Submitted on 10 Nov 2020

HAL is a multi-disciplinary open access archive for the deposit and dissemination of scientific research documents, whether they are published or not. The documents may come from teaching and research institutions in France or abroad, or from public or private research centers.
L'archive ouverte pluridisciplinaire HAL, est destinée au dépôt et à la diffusion de documents scientifiques de niveau recherche, publiés ou non, émanant des établissements d'enseignement et de recherche français ou étrangers, des laboratoires publics ou privés. 
Journal : Biometals (2019) 32:501-519

https://doi.org/10.1007/s10534-019-00201-8

Received: 11 May 2019 / Accepted: 23 May 2019 / Published online: 4 June 2019

New insights into the tetrameric family of the Fur metalloregulators

S. Nader, J. Pérard*, P. Carpentier, L. Arnaud, S. Crouzy*, I. Michaud-Soret ${ }^{\star}$

Univ-Grenoble Alpes, CEA, CNRS, BIG, CBM, CEA-Grenoble, 38000 Grenoble

serge.crouzy@cea.fr

julien.perard@cea.fr

isabelle.michaud-soret@cea.fr

phone numbers: 33438789940

https://orcid.org/0000-0002-8936-4964 


\section{Abstract}

The ferric uptake regulator (Fur) belongs to the family of the metal-responsive transcriptional regulators. Fur is a global regulator found in all proteobacteria. It controls the transcription of a wide variety of genes involved in iron metabolism but also in oxidative stress or virulence factor synthesis. As a general view, Fur proteins were considered to be dimeric proteins both in solution and when bound to DNA. However, our recent data demonstrate that Fur proteins can be classified into two subfamilies, according to their quaternary structure. The group of dimers is represented by E. coli, $V$. cholerae and $Y$. pestis Fur and the group of highly stable tetramers by $P$. aeruginosa and $F$. tularensis Fur. Here, another tetrameric structure of a PaFur mutant containing manganese and zinc metal ions is described. Through biochemical, structural and computational studies, we have deciphered the important structural characteristics of the tetramers and studied the main interactions responsible for their strength. Potential or mean force calculations for tetramer formation have been determinant to quantify these interactions. Moreover calculations allow us to propose that some conserved residues prevent the tetramerization in the subfamily of dimeric Fur.

Keyword: metal homeostasis structure of metalloregulators; quaternary structure; iron uptake; molecular interactions; potential of mean force; ferric uptake; tetramer.

\section{Acknowledgements}

We acknowledge the use of resources of INEXT 2217 and the support of members of the HTX Lab (EMBL, Grenoble, France). We also thank the European Synchrotron Radiation Facility for access to beamlines FIP BM30A and ID30A-1. This work was fund by the CEA, the Laboratory of Excellence GRAL (ANR-11-LABX-49-01) and the LabEx ARCANE and CBHEUR-GS (ANR-17-EURE-0003). S.N. was supported by the Region Rhône-Alpes (ARC santé).

\section{Author contributions}

J.P., S.C. and I.M.-S. designed the research; S.N., L.A. and J.P. carried out the biochemical and structural experiments, J.P. and P.C. resolved the structure; S.N. and S.C. did the theoretical work; J.P., S.N., S.C. and I.M.-S. analyzed data; S.N., J.P., S.C. and I.M.-S. wrote the paper.

Conflict of Interest: The authors declare that they have no conflict of interest. 


\section{Introduction}

Fur transcription factors sense iron status and control the expression of genes involved in iron homeostasis, virulence and oxidative stresses. Fur (Ferric uptake regulator) is an amazing multi-facet regulator, ubiquitous in Gram-negative bacteria (present in some Gram positive) and absent in eukaryotes. Due to the strong competition for iron acquisition between host and pathogens, Fur is a key protein involved in the bacterial virulence.

According to a dogmatic mechanism, a Fur dimer, activated by iron binding, controls the expression of genes involved in iron homeostasis and oxidative stress response. However, this regulation is not so simple since apo and holo activation and repression have been described, controlling iron transport, use and storage (Agriesti et al. 2014, Seo et al. 2014). Moreover, two dimers binds to the Fur box (Deng et al. 2015), which allows different levels of regulation, in time and as function of the $\mathrm{O}_{2}$ concentration and metal status of the growing environment (Beauchene et al. 2017, Pi \& Helmann 2017).

The oligomerization of Fur proteins has also been described from biochemical and biophysical studies (SEC-MALLS EcFur (D'Autreaux et al. 2007)) and several other Fur (Perard et al. 2016, Perard et al. 2018); footprinting (Delany et al. 2002); AFM and Electronic microscopy (Le Cam et al. 1994) and a role in DNA structure modification has been shown (stiffening (Le Cam et al. 1994)) and compaction (Roncarati et al. 2016).

Fur proteins contain mainly three types of metal binding sites. S1: an optional structural zinc site containing cysteine ligands stabilizing the dimer (sometimes replaced by a disulfide bridge or an ionic interaction in the case of PaFur); S2: considered as the regulatory site essential for the DNA binding activity for holo regulation; S3: optional, it has been proposed for tuning the regulatory strength as function of the metal status (Dian et al. 2011).

Several structures of Fur and Fur like proteins, in the apo, holo and DNA bound status have been described (Sarvan et al., 2018). They reveal common features: 1) a conserved DNA binding domain (DBD well structured and constituted of a winged helix turn helix motif; 2) a very flexible hinge between the DBD and the dimerization domain (DD); 3) a very likely structural Zn site S1 for dimer stabilization; 4) a S2 site required for DNA binding activation by the holo form; 5) different positioning of the DBD related to the DD with mainly inside or outside $\beta 1 \beta 2$ wings in absence of DNA and inside wings when bound to DNA.

However, some proteins like PaFur (Perard et al. 2016); and FtFur (Perard et al. 2018) are highly stable as tetramers in solution. In the case of FtFur, it has been shown that iron metallated tetramers could exist in vivo. The partition between dimers and tetramers in resolved protein structures in the PDB is shown in Supplementary Table S1. 
Fur from $E$. coli shares 53.7 and $38.6 \%$ sequence identity with $P$. aeruginosa and $F$ tularensis respectively and Fur from $P$. aeruginosa and F. tularensis $41 \%$ (Clustal and (Perard et al. 2016)). Even if EcFur is well characterized as a dimer, no X-ray diffraction structure of the whole protein is currently available. However, it shares $77 \%$ identity ( $91 \%$ homology) with VcFur whose structure has been described (Sheikh \& Taylor 2009).

The structure of PaFur was first described by Pohl as a dimer (Pohl et al. 2003) but our team demonstrated that it is a tetramer in solution and found out, using the PISA server (Protein Interfaces Surfaces and Assemblies) that Pohl's PDB structure was most probably a tetramer (Perard et al. 2016).

Another tetrameric structure of a PaFur mutant containing manganese and zinc metal ions will be described here. In this work, we deciphered the important structural characteristics of the tetramer and studied the main interactions responsible for its strength. One of the intriguing features is that, in absence of metal ions or in presence of metal ions but in absence of DNA, these apo and metalled proteins are still tetrameric. Consequently, the determinants of tetramer stability should be present in the sequence. Based on this fact, we performed a simple phylogenetic study to detect a possible evolutionary link between oligomeric states of Fur sequences and species kinship. We completed our experimental biochemical and structural studies with a theoretical work to investigate the reason behind these different quaternary structures of Fur proteins. In silico dissociation experiments have been performed to compare the strength of interactions between different physiological tetramers or modelled ones through the calculation of potentials of mean force and to decipher the most important residues involved in the dimer/dimer interactions. 


\section{Results}

\section{Alignment and Phylogenic tree}

As the protein folding depends on the sequence, the first steps in our study consisted of grouping Fur proteins using their amino acid sequences. The bacterial species were chosen to represent different bacterial phyla and classes in addition to species where Fur proteins were structurally characterized, or extensively studied. The phylogenetic tree generated by the Maximum Likelihood method (Guindon et al. 2010) using the Seaview software (Gouy et al. 2010) for bacterial species depending on their Fur amino acid sequence is shown in Figure 1 (and the alignment with Seaview in Supplementary Figure S1). Tetrameric Fur proteins from L. pneumophilia, $P$. aeruginosa and $F$. tularensis are shown in green. Dimeric Fur proteins are shown in orange. Interestingly, tetrameric Fur proteins are grouped together indicating that their oligomeric state is based on shared residues between the three Fur proteins.

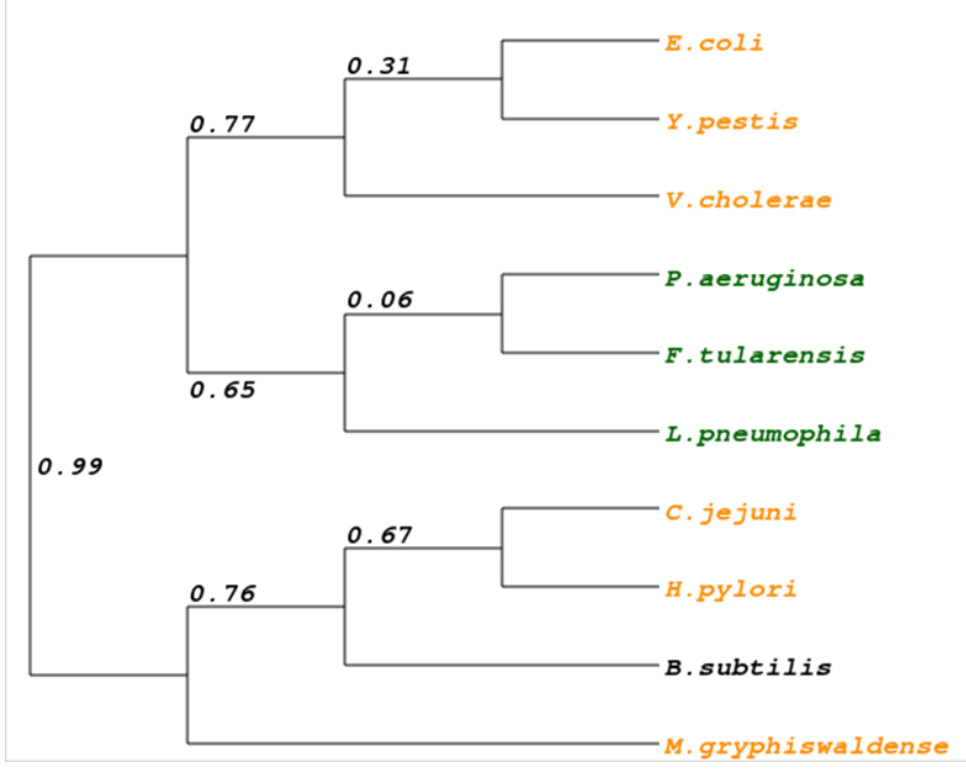

Fig. 1: Phylogenetic tree of Fur proteins with known oligomeric states from biochemical studies. In order to generate the tree, alignments (Figure S1) were restricted to the common part of the sequence (cut in N-ter and C-ter). Tetrameric Fur proteins are shown in green, dimers are shown in orange. $B$. subtilis is the only Gram-positive strain

From the alignment itself, there is no obvious partition of amino acid sequence between dimers and tetramers. We will discuss the sequence comparison later on together with the simulation results about important residues involved in the dimer-dimer interactions.

Structural analysis of FtFur and PaFur and simulations were done to understand how tetramers dissociate into dimers that bind DNA. 


\section{Structure of the tetramer of Fur H86A-H124A from P. aeruginosa (PaFur-mS3), an active}

\section{mutant with a residual S3 site}

The structure of PaFur was the first Fur to be described in the literature by Pohl et al (Pohl et al. 2003). The structure of the subunit of PaFur-WT in the PDB (PDB ID: 1MZB) and its description as a dimer are shown in Figure $2 \mathrm{~A}$ and B. However, We later showed that this protein is tetrameric in solution and predicted as a tetramer by the PISA software (Perard et al. 2016).
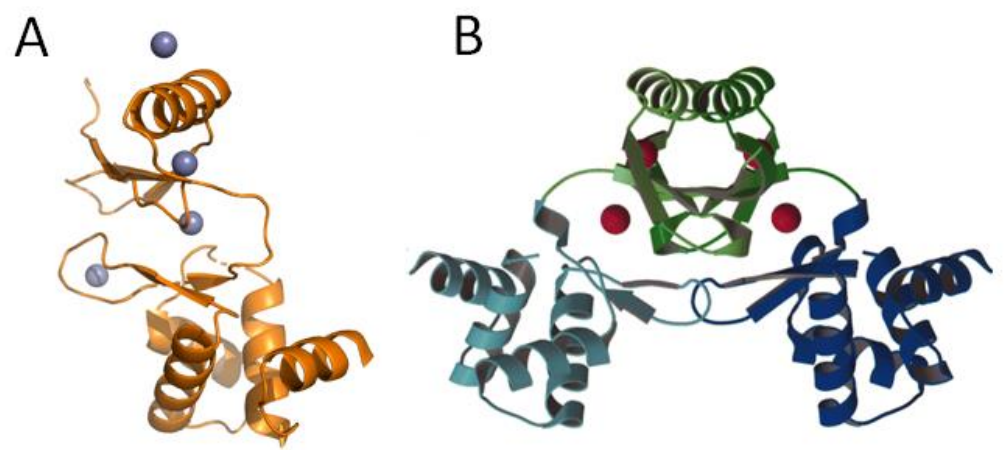

Fig. 2: A) Structure of PaFur-WT (PDB ID: 1MZB (Pohl et al. 2003)) showing one Fur subunit and $4 \mathrm{Zn}^{2+}$ atoms. B) Structure of the dimer as described by Pohl (Pohl et al. 2003) showing 2 $\mathrm{Zn}^{2+}$ per subunit.

We purified and biochemically characterized the PaFur tetramer which does not contain any metal ions when purified (Perard et al. 2016) but we were not able to reproduce the crystals of PaFur wild type described by Pohl et al (Pohl et al. 2003). However good diffracting crystals of PaFur H86A-H124A mutant (PaFur-mS3) were obtained and the structure was solved (Table1 and Figure S3). The goal was to study the impact of the S3 site by mutating these two residues. This mutant is still a very stable tetramer in solution and can be activated by manganese dications for its specific DNA binding as the wild type protein, but not by zinc (Figure S2). Its structure was obtained in presence of $\mathrm{Mn}^{2+}$ and $\mathrm{Zn}^{2+}$ at $2.34 \AA$ resolution (PDB code $6 \mathrm{H} 1 \mathrm{C}$ ). The structure, shown in Figure S3, in contrast to the structure described by Pohl, consists of two Fur subunits belonging to different dimers. A tetramer can be reconstructed from the electron density with four Fur subunits belonging to two different meshes (Figure S3B). 


\begin{tabular}{|c|c|c|}
\hline Sample name & \multicolumn{2}{|c|}{ Mn-PaFur_mS3 } \\
\hline \multicolumn{3}{|l|}{ Data collection } \\
\hline pdb deposition code & \multicolumn{2}{|c|}{$6 \mathrm{H} 1 \mathrm{C}$} \\
\hline Beamline & \multicolumn{2}{|c|}{ id23eh1 (ESRF) } \\
\hline Space group & \multicolumn{2}{|c|}{$\mathrm{P} 66_{1} 22$} \\
\hline \multicolumn{3}{|l|}{ Unit cell dimensions: } \\
\hline a, b, c $(\AA)$ & 85.5785 .57179 .62 & 85.0585 .05177 .46 \\
\hline$\alpha, \beta, \gamma\left(^{\circ}\right)$ & $90.00,90.00,120.00$ & $90.00,90.00,120.00$ \\
\hline Wavelength $(\AA ̊)$ & 0.9797 (remote) & 1.2925 (peak) \\
\hline Resolution range $(\AA \AA)$ & $68.5-2.34(2.42-2.34)$ & $42.52-2.61(2.27-2.61)$ \\
\hline R-merge & $0.038(0.17)$ & $0.037(0.17)$ \\
\hline R-meas & $0.05(0.24)$ & $0.06(0.24)$ \\
\hline Total reflections & 33998 (3342) & 20008(1923) \\
\hline Unique reflections & $17023(1671)$ & $15650(1540)$ \\
\hline Mean I/sigma(I) & $14.12(3.99)$ & $10.2(1.60)$ \\
\hline Completeness (\%) & $99.22(99.94)$ & $99.72(99.07)$ \\
\hline $\mathrm{CC}(1 / 2)$ & $99.8(47.6)$ & $99.5(57.8)$ \\
\hline Redundancy & $2.1(2.0)$ & $1.8(1.7)$ \\
\hline \multicolumn{3}{|l|}{ Anomalous } \\
\hline$f^{\prime \prime}(Z n)$ & $2.5 \mathrm{e}$ & $0.5 \mathrm{e}$ \\
\hline $\mathrm{f}^{\prime \prime}(\mathrm{Mn})$ & $1.3 \mathrm{e}$ & $2.1 \mathrm{e}$ \\
\hline \multicolumn{3}{|c|}{$\begin{array}{l}\text { Integration statistics from XDS/XSCALE; * Redundancy independent R-factor (intensities); } \\
\text { Values in parentheses are for highest-resolution shell }\end{array}$} \\
\hline \multicolumn{3}{|l|}{ Refinement } \\
\hline Resolution range $(\AA ̊)$ & $68.5-2.34$ & \\
\hline Reflections used in refinement & 17023 & \\
\hline Reflections used for R-free & 804 & \\
\hline R-work (\%) & 20.1 & \\
\hline R-free (\%) & 26.8 & \\
\hline Wilson B-factor & 35.26 & \\
\hline \multicolumn{3}{|l|}{$N^{\circ}$ atoms: } \\
\hline total & 2217 & \\
\hline macromolecules & 2107 & \\
\hline ligands & $23(12 \mathrm{Zn}+11 \mathrm{Mn})$ & \\
\hline Water & 106 & \\
\hline Protein residues & 266 (132 in chain $A+134$ chain $B)$ & \\
\hline RMS(bonds) & 0.008 & \\
\hline RMS(angles) & 0.86 & \\
\hline \multicolumn{3}{|l|}{ Ramachadran: } \\
\hline favored (\%) & 95.06 & \\
\hline allowed (\%) & 3.8 & \\
\hline outliers (\%) & 1.14 & \\
\hline Rotamer outliers (\%) & 6.82 & \\
\hline \multicolumn{3}{|c|}{ B-factors $\left(\AA^{2}\right)$ (calculated by CCP4) } \\
\hline average & 37.1 & \\
\hline macromolecules & 35.3 (chain A) 36.6 (chain B) & \\
\hline ligands & 50.3 -> $43.2(\mathrm{Mn}), 65.5(\mathrm{Zn})$ & \\
\hline solvent & 37.3 & \\
\hline
\end{tabular}

Table 1 Data collection, phasing and refinement statistics for the structures of Mn-PaFur-mS3. Values in parentheses are for highest-resolution shell. Integration statistics are from XDS/XSCALE (Kabsch 2010), refinement statistics are from Phenix (Adams et al. 2010) 
In Fig.3, we show a detailed view of the structure with its metal sites, metal atoms and water molecules while keeping the same orientation as the density map (Figure S3). The structure is similar in secondary and tertiary structure to the known Fur structures previously described. However, interesting features appear as to the number of bound metal cations and corresponding metal sites. They will be described in more detail in the following.

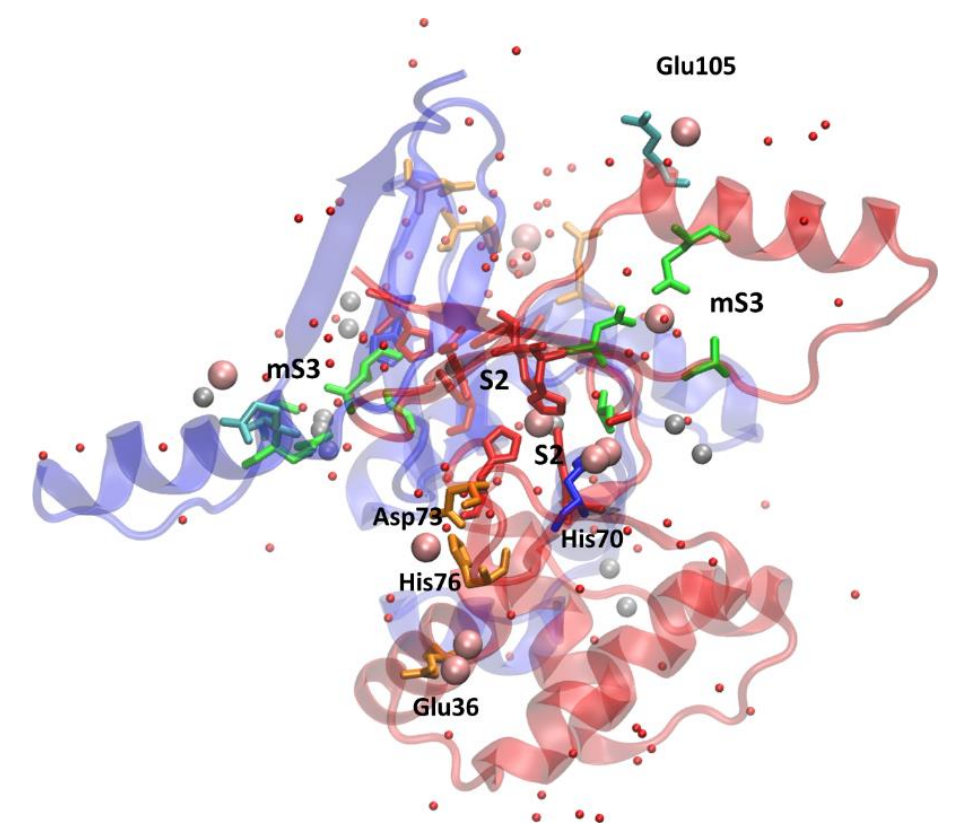

Fig. 3: Mn-PaFur-mS3 as seen in the crystallographic asymmetric unit (PDB 6H1C) in the same orientation as Fig. S3B. $\mathrm{Zn}^{2+}$ and $\mathrm{Mn}^{2+}$ cations are represented as grey and pink spheres, respectively. The S2 and S3 conventional metal sites are highlighted in red and green, respectively. In our structure, $\mathrm{Mn}^{2+}$ ions in non-conventional sites are coordinated by His70 in blue, Glu 105 in cyan, Glu36, Asp73 and His76 in orange. Some sites are too close to each other to coexist in a same structure and they should be considered as variations of the metal ion positioning in one low affinity site. Water molecules are represented by small red spheres.

The structure of PaFur-mS3 from $P$. aeruginosa was resolved with $12 \mathrm{Zn}^{2+}$ atoms and $11 \mathrm{Mn}^{2+}$ atoms (Table 1). The structure has been analysed to investigate these unconventional metal sites (other than sites S2 and S3). Interestingly, the protein was thoroughly washed before crystallization with the same protocol performed previously for Fur from Francisella tularensis, which only contained metal filled S1 and S2 metal binding sites. The presence of multiple metal binding sites in PaFur-mS3 raises the question about a possible role in metal storage.

\section{S2 conventional metal sites of PaFur-mS3}

The S2 site found in the new structure is similar to the one described in PaFur-WT, with the same ligands His32, Glu80, His89 and Glu100 (Fig. 4 top left). 


\section{S3 metal sites of PaFur-mS3}

The S3 site is formed by His86, Asp88, Glu107 and His124 in the wild type protein as shown in Figure 4 (bottom left). In our mutated PaFur-mS3 (H86-A, H124A) construction, the residual S3 site only contains Asp88 and Glu107 as seen in Figure 4 (bottom right). The coordination sphere is completed with a water molecule.

PaFur-mS3 is still active when manganese ions are added and since this residual S3 site could not be a high affinity site, we can conclude that $\mathrm{S} 2$ is the regulatory site, similarly to the other well characterized Fur proteins such as HpFur(Dian et al. 2011) and in contrary to Pohl's conclusions.
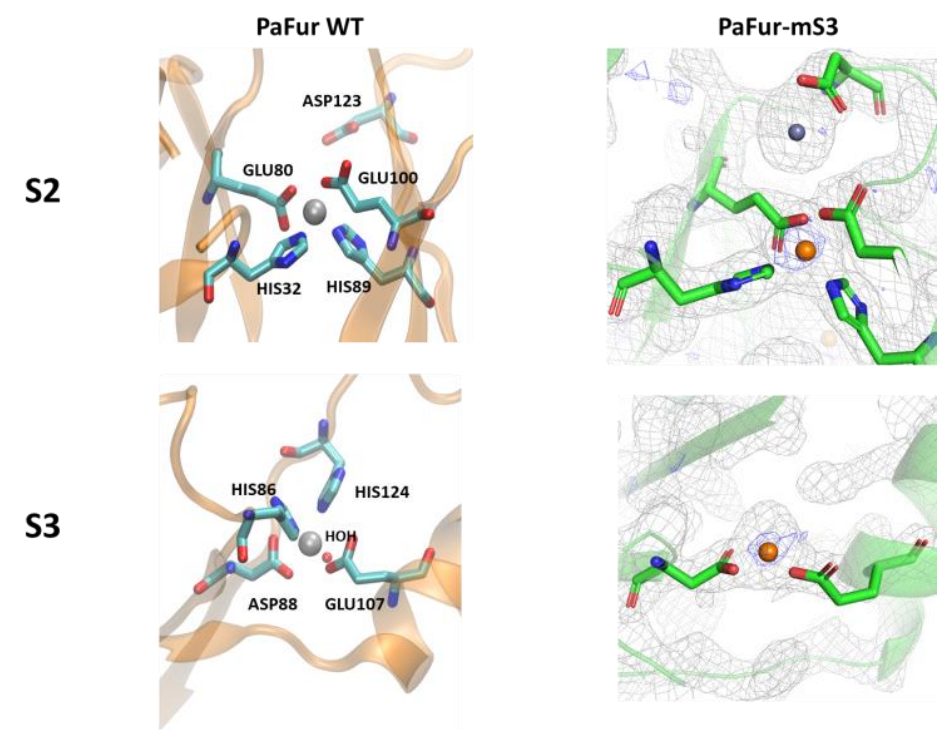

Fig. 4: Comparison of the chemical environment of $S 2$ and $S 3$ sites in the PaFur-WT structure containing zinc (Pohl et al., 2003 - Left) and in PaFur-mS3 (right) containing manganese ions. Electron density maps are shown with unambiguous positioning of the $\mathrm{Mn}^{2+}$ atoms in the anomalous map at $1.29 \AA$ (figure S3). Due to the mutations, the residual S3 site only has Asp88 and Glu107 as ligand in addition to water molecules.

\section{Supplementary lower affinity nonconventional metal sites}

Our biophysical characterization of PaFur-WT and PaFur-mS3 indicates that they are tetramers in solution (Perard et al. 2016). In order to study the physiological oligomeric state, the crystal symmetries were used to generate a PaFur-mS3 tetramer since the tetramer was visible in the electron density map (Figure S3). 
a) PaFur-WT

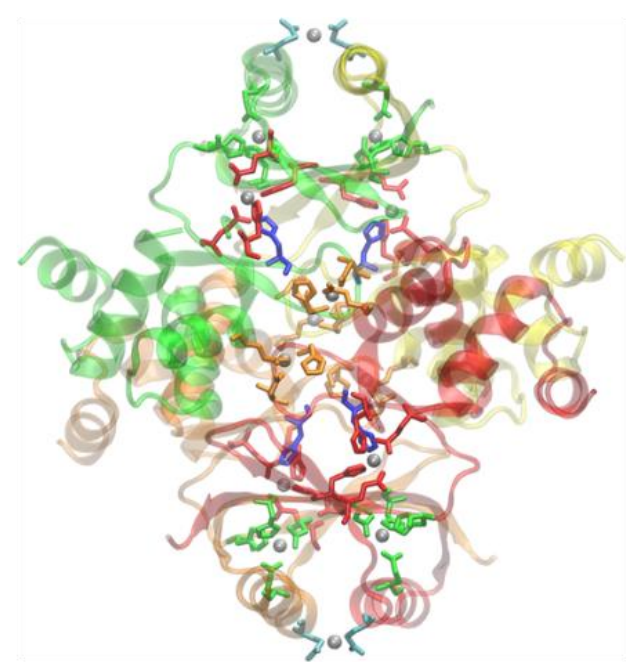

b) PaFur-mS3

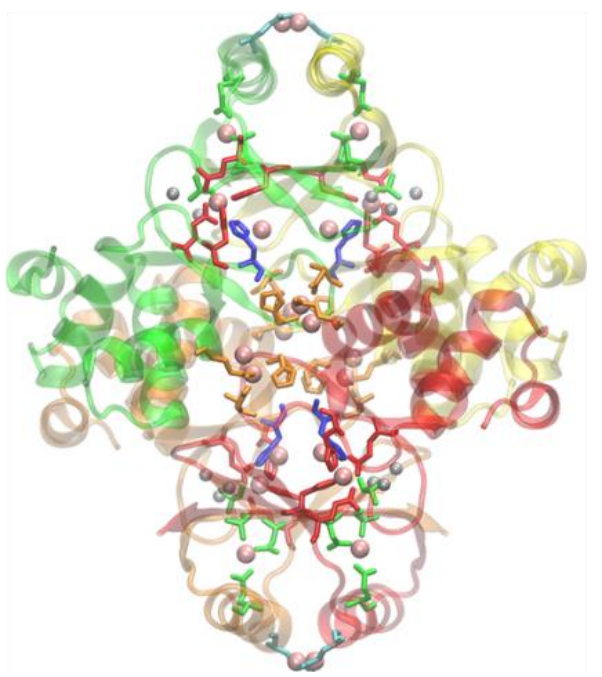

Fig. 5 Zn-PaFur from Pohl's 1MZB (a) and Mn-PaFur-mS3 (b) tetramers generated by symmetry. $\mathrm{Zn}^{2+}$ and $\mathrm{Mn}^{2+}$ cations are represented as grey and orange spheres, respectively. The S2 and S3 conventional metal sites are highlighted in red and green, respectively. In our structure, $\mathrm{Mn}^{2+}$ ions in non-conventional sites are coordinated by His70 in blue, Glu 105 in cyan, Glu36 Asp73 and His76 in orange. (Chains A, B, C and D are represented in green, yellow, red and orange, respectively).

From both the crystal structure and the tetramer constructions shown in Fig. 5, several "unconventional" metal sites appear (some of them seen in the wild type structure by Pohl). They could possibly be low affinity sites, involved in the protein packing during crystallization, even after thoroughly washing the protein before crystallization:

- His70 coordinates one $\mathrm{Mn}^{2+}$ ion.

- Two Glu105 from two different subunits (and two different asymmetric units) coordinate one $\mathrm{Mn}^{2+}$ ion (one $\mathrm{Zn}^{2+}$ ion in Pohl's structure). In Mn-PaFur-mS3, two metal ions found on the symmetry axis are unrealistically close in our structure meaning that there should probably be only one of them really present in solution.

- Finally, Asp73, His76 from one subunit and Glu36, from another subunit in the other dimer, form another $\mathrm{Mn}^{2+}$ binding site found both in PaFur-WT filled with zinc and in PaFur-mS3 filled with manganese. Again, two manganese ions are seen in the structure, which may correspond to two alternative conformations with the same metal binding ligands. The manganese involved in most interactions with proteins ligands is 
shown in Figure 6. Interestingly, this last type of site links two dimers at the tetramer interface.

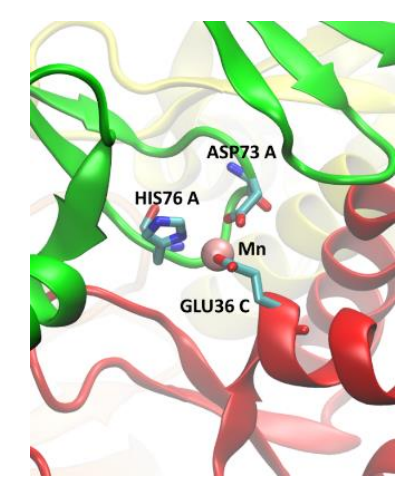

Fig. 6: Close view on the Mn-PaFur-mS3 structure showing an additional manganese binding site formed by residues from two subunits from different dimers. Here: His76 Asp73 from subunit $A$ in the $A-B$ dimer and Glu36 from subunit $C$ in the $C-D$ dimer.

Hence, in addition to the conventional S2 and S3 metal sites, the tetramers show that some metal sites can form between dimers and may be related to tetramer stability even though the purified protein does not contain any metal ion as purified. After treatment of PaFur-WT with 0.1M EDTA overnight at room temperature, the protein was still tetrameric in solution (Perard et al. 2016) indicating that these unconventional sites are not essential to the tetramer stabilization. A particularly interesting interaction involves one Glutamate 36 from one subunit of one dimer interacting with one metal ion also bound to two residues of one subunit of the other dimers, Aspartate 73 and Histidine 76. This site was found both in PaFurWT (Pohl) and PaFur-mS3 structures (this work) (Figure 5). In the absence of metal, H-bonds between these residues may exist. These sites were not found in the FtFur tetrameric structure where the region D73 to $\mathrm{H} 76$ is not close enough to D37 (corresponding to E36 in PaFur) to form a metal binding site. Furthermore, the H76 from PaFur is not conserved in other tetramers such as FtFur where there is a E76 Q77 sequence there.

In the PaFur-mS3 structure, $\mathrm{Mn}^{2+}$ replaces some of the $\mathrm{Zn}^{2+}$ atoms coming from the purification stage, in addition to populating several other sites. A similar behavior is expected for $\mathrm{Fe}^{2+}$ replacing $\mathrm{Zn}^{2+}$ when bound to PaFur in vivo. Taken together, these observations about unconventional metal sites may indicate a role of Fur protein as iron storage proteins buffering the cytosol metal content just as a metallothioneins or Hpn proteins do. The affinity of the metal for these unconventional metal sites should be analyzed in the future as well as their relevance in vivo. 


\section{Sequence analysis of the differences between dimers and tetramers}
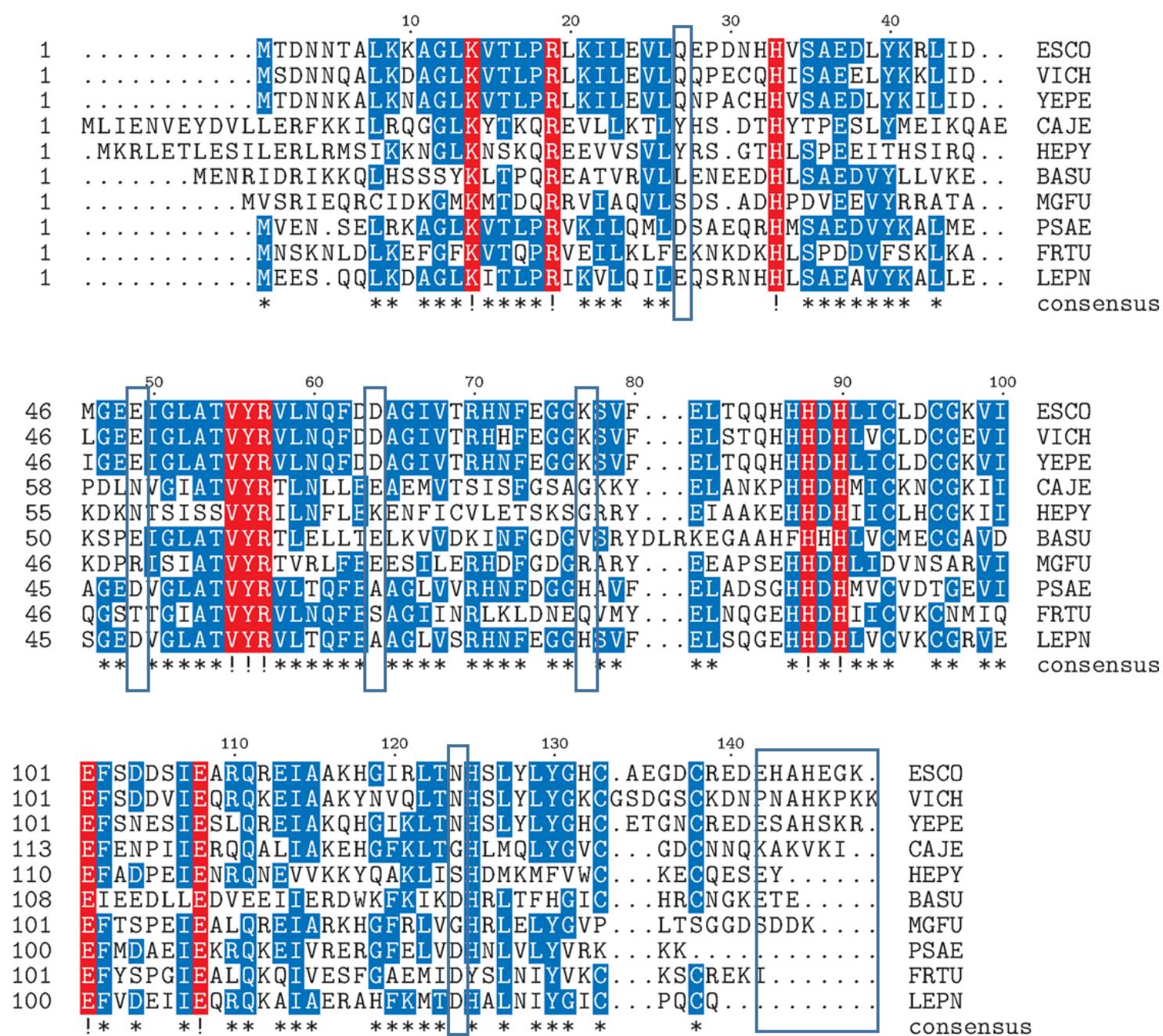

Fig. 7: Sequence alignment of Fur proteins studied in this work. Escherichia coli(ESCO), Vibrio cholera(VICH), Yersinia pestis(YEPE), Campylobacter jejuni(CAJE), Helicobacter pylori(HEPY), Bacillus subtilis(BASU), Magnetospirillum gryphiswaldense(MGFU), Pseudomonas aeruginosa(PSAE), Francisella tularensis(FRTU) and Legionella pneumophila(LEPN). The framed residues are different between dimers and tetramers. The coloring scheme stands for completely (red) or partially (blue) conserved residues.

Several residues have already been identified as important for Fur activity and are conserved within the Fur family: this is the case of Lys 14, Arg 19, Thr 54, Val 55, Tyr 56, Arg 57, and Glu 101 (using $E$. coli or $V$. cholerae Fur numbering). The largely conserved sequence of residues 87-90, His 33 and Glu 108 (EcFur $n^{\circ}$ ) contains histidine and acidic residues involved in the formation of the S2 and S3 metal sites.

Concerning the distinction between dimers and tetramers, it is noticeable that the dimeric proteins seem to all have a longer $\mathrm{C}$-ter, which was not resolved in the structures and then not used in the simulations. However, we know that $\Delta$ C-ter $E$. coli Fur (1-140) is still an active dimer in solution (data not shown). 
Other differences are in positions 27, 49, 64, 77 and 124 (using E. coli or V. cholerae Fur numbering) as highlighted in Figure 7.

At position 27, tetramers contain acidic residues whereas dimers do not. In position 49, tetramers contains mainly $\mathrm{D}$ and dimers mainly $\mathrm{E}$.

One noticeable difference of charge is seen in the sequence F62 E63 X64. The sequence FEA or FES seems to be present in all Fur proteins forming tetramers whereas an aspartic $(X=D$ or $\mathrm{E}$ ) is seen in Fur proteins forming dimers. Two glutamate residues $\mathrm{E} 63$ in $\mathrm{Ft}$, (E62 in $\mathrm{Pa}$ ) make two salt bridges with arginine R57 in Ft, (R56 in Pa-mS3). This salt bridge is known to be implied in the stabilization of the tetramer of FtFur and could be important in its dissociation and binding of the dimer to the Fur box after interaction of the arginine with DNA. Other differences may play a role: $\mathrm{A}$ or $\mathrm{Q}$ in tetramers replaces a positively charged residue around position 77 in dimers. More subtle differences are visible in the C-terminal part of the sequences: $\mathrm{D}$ residue conserved at position 124 in tetramers but not in dimers where a $\mathrm{N}$ is mainly present (except in BsFur CjFur, MgFur and HpFur where there is a D, G, G or $S$ respectively). Sequences differences implied in the distinction dimer/tetramer will be further discussed in the light of the molecular dynamic results.

\section{In silico dissociation studies}

To understand how tetramers dissociate and bind DNA as dimers, simulations of tetramer complexes were carried out to find which complex is more stable and what initiates their dissociation. This was done by calculating the free energy profiles of the dissociation process and major interacting residues in each case. FtFur, PaFur and PaFur-mS3 real tetramers were compared to an in silico designed model of a VcFur tetramer. VcFur is described as a dimer and is very close in sequence to EcFur which has been shown to be mainly a dimer in solution with the presence of tetramers at high concentration (estimated Kd $1 \mathrm{mM}$ ) (D'Autreaux et al. 2007)). The different structures and models are shown in Figure 8a and the potentials of mean force for the dissociation of all the tetrameric complexes that we simulated are shown in Figure 8b. Calculations were carried on until good convergence and reliable error estimation could be obtained. Statistical errors were estimated to be $<1.5 \mathrm{kcal}^{\mathrm{mol}}{ }^{-1}$ with bootstrap analysis using the 'Bayesian bootstrap' method(Efron 1979) (b-hist option in g_wham). After 60 ns simulation time per window, the binding free energies from dimer to tetramer were estimated and presented in Figure 8c. (The corresponding error bars are shown in Supp Fig. S5). The profiles show a slightly more stable PaFur-WT tetramer compared to PaFur-mS3 by $1.25 \mathrm{kcal}^{\mathrm{mol}}{ }^{-1}$. Interestingly, the binding $\Delta \mathrm{G}$ of FtFur tetramer is lower than that of PaFur by a significant 3 $\mathrm{kcal} \mathrm{mol}^{-1}$. The crystal structures of PaFur-mS3 show more metal atoms bound to the protein 
than in the case of FtFur. This can be one possible explanation for the different behavior observed here.

In the case of the VcFur tetramer, the fact that the minimum of the free energy profile is found at a center of mass- center of mass distance of 27 to $28 \AA$ instead of 21 to $22 \AA$ for other tetramers, could be due to a bad packing of the loops between the dimerization domain and the DNA binding domain. This region extends more easily during the pulling, leaving the moving DBD less affected by the translation and thus likely to maintain their interactions with the fixed DBD, or even enhance them. However, this complex has the lowest $\Delta G$ of binding and validates, as a negative control, our simulation protocol.

a)

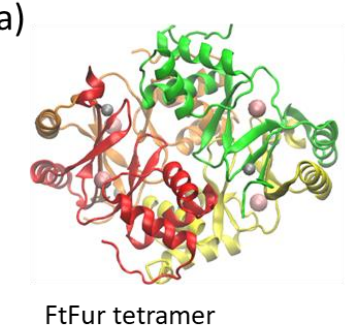

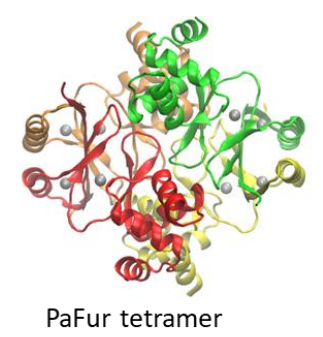

)

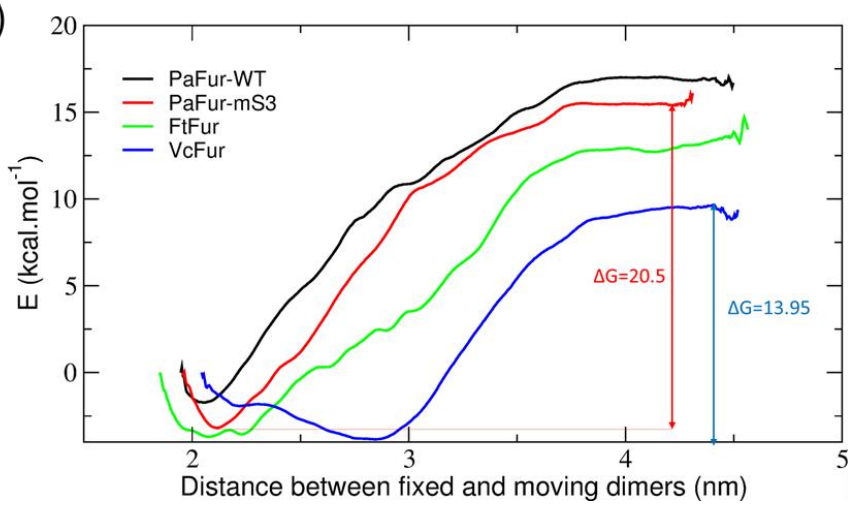

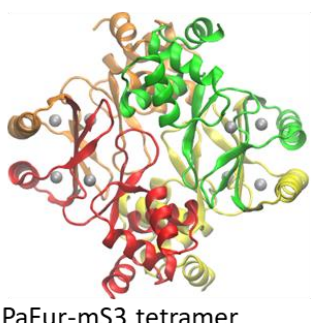

PaFur-mS3 tetramer

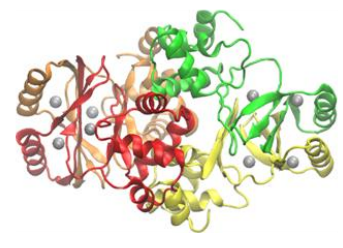

built VcFur tetramer

c)

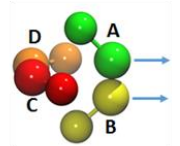

\begin{tabular}{|l|c|}
\hline Tetramer & $\boldsymbol{\Delta} \mathbf{G}\left(\mathrm{kcal}_{\mathrm{mol}} \mathbf{m}^{-1}\right)$ \\
\hline PaFur-WT & $19.25 \pm 2.2$ \\
\hline PaFur-mS3 & $20.50 \pm 1.7$ \\
\hline FtFur & $16.71 \pm 1.7$ \\
\hline VcFur & $13.95 \pm 2.3$ \\
\hline
\end{tabular}

Fig. 8: a) Calculated minimum energy structures of the tetramer complexes. Chains $A, B, C$ and $D$ are represented in green, yellow, red and orange, respectively. b) Plot showing the potential of mean force curves of the tetrameric Fur complexes; c) Computed binding free energies $(\Delta \mathrm{G})$ from dimers to tetramers of Fur proteins

From our calculations, interaction energies, sums of the electrostatic and van der Waals (Lennard Jones potential) energies can be estimated (See Methods). By calculating the average individual interactions energies between each residue for a moving chain and the fixed dimer, the total interaction energy of that chain can be determined. When this total interaction energy is plotted against distance, the different behaviors of the moving chains during the dissociation can be compared. From the plots shown in Supplementary Figure S6, the two chains seem to interact similarly with interactions with the fixed dimer decreasing with 
distance, indicating that most probably, a symmetrical dissociation is observed, since both chains should have a similar set of interacting partners within the tetramer. This idea of symmetric dissociation relies on large interaction energies calculated at short distances when the tetramers are formed and stable. We will emphasize, on the contrary, in the next section, an asymmetry in the first step of association between the dimers at long distances.

Looking at interactions of individual residues in a chain throughout the whole simulation in Figure S7, 15 common residues between chain A and B can be determined. Other than displaying the contributions of major interacting residues in comparison with the total interaction energy, and comparing residues with each other's, this type of visualization gives a detailed idea about the evolution of individual interactions during the dissociation of the system.

Translated into numbers, the plots shown in Figure S7 give Table S3 in the supplementary information section, where only the top 5 interacting residues, of each chain, are detailed.

Since we are simulating a dissociation, by inverting the process we can gain insights into the complex formation. Three main types of residues can be distinguished:

- Residues present from the start to the end of the simulation, could be important for recognition, initiation of oligomerization or DNA binding and involved in locking the complex in its final, bound, state.

- Residues present at the start but fading out before the end of the simulation, could be important for locking the final conformation in place. Theoretically, this type of residues should be found on the inner surface of the interaction area.

- Residues absent at the start but present at the end of the simulation could be important for the first series of recognition interactions, initiating complex formation.

\section{Results from early stages of the interactions during the dynamics}

Analyzing the interacting residues along the dynamics of interaction of both native $\mathrm{Ft}$ and $\mathrm{Pa}$ tetramers and in silico-model Vc tetramer, we hope to decipher the clues responsible for the stability of the two oligomeric state families of Fur. Not surprisingly, all the interactions during the approach of the two dimers involve the DBD residues. Interactions involving residues 5 to 80 of all Fur tetramer models in windows 16 and 20 are shown in Figure 9 (windows 9 and 18 are also presented in Figure S9 together with the full data for PaFur). 


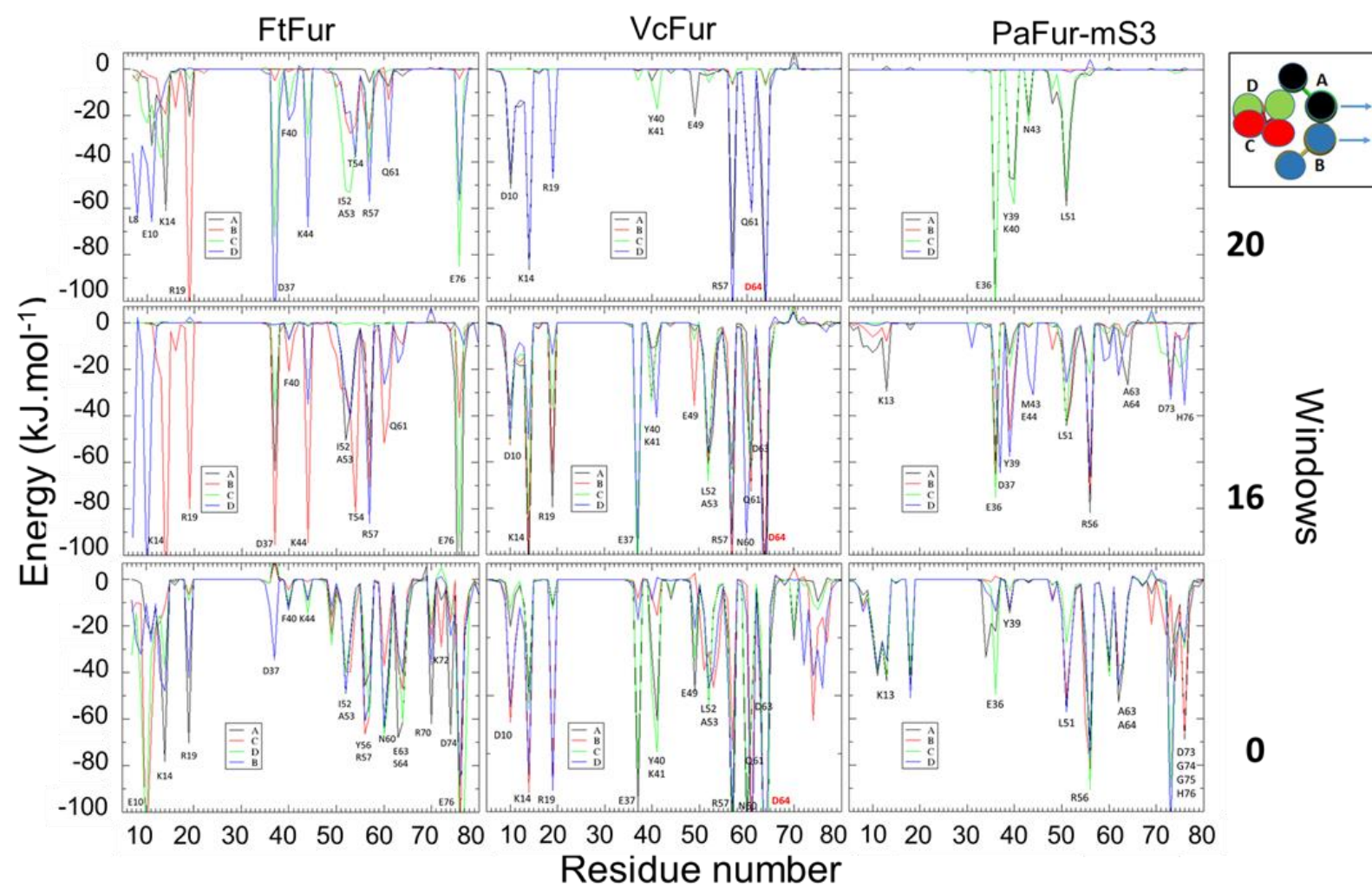

Fig. 9: Average interaction energies calculated from the $\approx 50$ ns umbrella sampling $M D$ simulations used to create the potentials of mean force for three different windows. The contributions of each of the four subunits in the tetramer are shown in different colors. For clarity and homogeneity, energies are displayed between -100 and $+7 \mathrm{kcal}^{\mathrm{mol}}{ }^{-1}$ and residue numbers limited to the range $[5,80]$. Apart from slightly different positioning of the proteins PaFur-ms3 and PaFur give similar results and the latter is not shown.

A detailed description of the interactions between dimers during their approach is given below for each Fur model:

VcFur

For VcFur, the analysis of the overall energy per residue along the whole process of association yields the most important residues, such as D64, E37, Q61, K14 and D10 (Fig. 9). One of them, D64 is very interesting because it has a very important contribution in energy and no homolog in the sequences of the "true" tetramers where the corresponding residues are A or S. Looking at its energy as a function of time during the approach of the two dimers, (represented by the dynamics windows), we observe a large contribution change between windows 16 and 20. Indeed, the representation of the energy per residue at windows 16,18 and 20 (meaning when the two dimers begin to interact) is very informative. At window 20 , all 
the interactions involve moving chain A and fixed chain D: D64 with Q61 (R19 and R57), R19 with D64; K14 with D10 and R57 with D64 (and N60). The approach is asymmetric with almost all interactions involving only these two chains.

When distances are reduced in windows 18 ( $2 \AA$ closer between the two dimers) and window 16 ( 4 A closer), E37 appears with high interaction energy with the backbone amines of residues G51, L52 and A53. Furthermore, the strong interaction energies of D64 now involve the 4 chains (two dimers) with equal importance with emphasis on the interactions with R19 and Q61. The energy of R19 increases for chains BC and decreases for chain D. The Y40 and K41 energies start to increase for the 4 chains, as well.

FtFur

At window 20, many interactions involve moving chain B and fixed chain D: K44 with D37 and E76, E76 with R57; few interactions involve fixed chain $C$ with a large interaction energy for $R 19$ with carbonyl of $F 11$ in chain $B$ which decreases in windows 18 and 16 while an interaction between $\mathrm{K} 14(\mathrm{C})$ and $\mathrm{E} 10(\mathrm{~B})$ increases.

Note that R19 and K14 are fully conserved residues known to be important for DNA interaction (base-specific for K14 with the Fur box).

The amino acid sequence from L71 to M79 is specific to FtFur. Hence, stabilizing interactions exist between E76 and N60, D74 and Q77 or Q77 and N97 through H-bonds not seen in the other tetramers.

PaFur and PaFur-mS3

In PaFur window 20, the largest interaction energy involves E44 a glutamate in the $\alpha 3$ helix (Ser34 to Ala45). E44 (C) interacts with backbone amines of L51 and A52 (A).

In PaFur-mS3 (Figure 9), due to a slightly different positioning of the $\alpha 3$ helices, E36 $(A, C)$ plays the role of E44 and interacts strongly and symmetrically with $\mathrm{Y} 39(\mathrm{C}, \mathrm{A})$ and backbone amine of $L 51(C)$ while $K 40(C)$ interacts with backbone of M43 (A).

Interactions between E36 and L51 and A52 become prominent in the two proteins when the dimers are moved closer from one another.

Interestingly, starting at window 18, R56 (D) interacts with carbonyls of G74 pointed out as important in PaFur-mS3 and the conserved G75 (B).

Not surprisingly, the first interactions involve the $\alpha 3$ and $\alpha 4$ helices (Gly50 to Ala64) residues. In windows 16, interactions between R56 (D) and D73 (B) and H76 (D) with E36 (B) appear for both proteins. D73 and $\mathrm{H} 76$ are both located in the loop between the first and second $\beta$ sheets. In the X Ray structures the E36, D73 and H76 residues stabilize the tetramer in presence of metal ions where they are part of an extra metal binding site able to bind $\mathrm{Zn}^{2+}$ (PaFur) or manganese (PaFur-mS3) (See Fig. 6). In our simulations, in the absence of extra metal binding sites, $\mathrm{H} 76$ and E36 probably also stabilize the tetramer due their direct interaction. 


\section{Comparison between Fur proteins}

Compared to $\mathrm{Vc}$ and FtFur, there is almost no interaction between $\mathrm{N}$-ter residues from two subunits of different dimers in both $\mathrm{Pa}$ and $\mathrm{Pa}-\mathrm{mS} 3$ because of the replacement of the negative $\mathrm{D} / \mathrm{E} 10$ in Vc and Ft (interacting with K14 and R19) by a K9.

An interaction between R57 and D74 (Ft) is present in both natural tetramers and not in the $\mathrm{Vc}$ tetrameric model (E74).

The above listed residues, which vary between dimer and tetramers (27, 49, 64, 77 and 124) have been followed along the dynamics. Interestingly, residue D64 already shows many more interactions in the VcFur model of tetramer when the two dimers are far apart (Figure S8). Residues 49 and 77 show similar medium interactions whereas residues 27 (not shown) and 124 show almost no interactions.

\section{Residues interacting in the crystallographic structure or last stage of the simulations.}

Interactions involving residues 5 to 80 of all Fur tetramer models in window 0 are also shown in Figure 9 and the results are summarized together with the interactions already present in the X-ray structures in Table S3. This study allows us to distinguish several interaction patches (Figure 10 and Table 2):

1- Interactions in the $\mathrm{N}$-terminal part of $A$ with $D$ and $B$ with $C$ subunits implying the highly conserved residues Lys (K14 in Ft and Vc, K13 in Pa) and D10 in Vc or E10 in Ft and the carbonyl of G11 in Pa-mS3. interactions between N-ter K14, R19 of Vc tetrameric model with D64 in the recognition helix do not exist in the real $\mathrm{Pa}$ and Ft tetramer which do not contain an acidic charged residues in that position (Figure 10 in pink).

2- Several interactions between residues of the DNA recognition helices (52-65 in Ft 5164 in $\mathrm{Pa}$ ) of $A$ with $D$ and $B$ with $C$ subunits (Figure 10 in green).

3- Interactions between the backbone of hydrophobic residues of the beginning of the recognition helices (51 in $\mathrm{Pa}$ and 52 in $\mathrm{Ft}$ ) and the backbone of the residues following C93 (Figure 10 in gray).

4- Interactions between D74 and R57 Ft (D73 and R56 Pa) are absent in VcFur model where the aspartate is replaced by a glutamate (Figure 10 in blue).

5- Finally, in Pa only, interaction of E36 with H76, through the manganese binding site in the metal containing Xray structure and with an $\mathrm{H}$-bond between those residues in the apo $\mathrm{Pa}$ (Figure 10 in cyan). 
These results are summarized in Table 2.

\begin{tabular}{|c|c|c|}
\hline FtFur & Pa Fur /PaFur-mS3 & VcFur \\
\hline$E 10 \rightarrow \mathrm{F} 11, \mathrm{~K} 14$ & K9 & $\mathrm{D} 10 \rightarrow \mathrm{K} 14$ \\
\hline $\mathrm{F} 11 \rightarrow \mathrm{K} 14$ & A10 & A11 \\
\hline$K 14 \rightarrow$ D7, K9, E10 & $\mathrm{K} 13 / \mathrm{K} 13 \rightarrow \mathrm{G} 11, \mathrm{~A} 63, \mathrm{~A} 64$ & $K 14 \rightarrow$ D10, D64, A65 \\
\hline$R 19 \rightarrow K 9, E 10$ & $\mathrm{R} 18 / \mathrm{R} 18 \rightarrow \mathrm{A} 63$ & $R 19 \rightarrow \mathrm{D} 64$ \\
\hline$D 37 \rightarrow 152$, A53 & $\begin{array}{l}\text { E36 /E36 } \\
\rightarrow \text { H76, L51, A52, G74 } \\
\rightarrow \text { L51, Y39, A52 (Zn/Mn) }\end{array}$ & $\begin{array}{l}\text { E37 } \\
\rightarrow \mathrm{G} 51, \mathrm{~L} 52, \mathrm{~A} 53, \mathrm{Y} 56\end{array}$ \\
\hline$F 40 \rightarrow 152$ & Y39 / Y39 $\rightarrow$ E36, Y39, D94, T95 & Y40 \\
\hline S41 & $\mathrm{K} 40 / \mathrm{K} 40 \rightarrow \mathrm{Y} 39, \mathrm{M} 43$ & $\mathrm{~K} 41 \rightarrow \mathrm{Y} 40, \mathrm{E} 49$ \\
\hline$K 44 \rightarrow \mathrm{D} 37$ & $\mathrm{M} 43 / \mathrm{M} 43 \rightarrow \mathrm{K} 40$ & 144 \\
\hline T49 (backbone) $\rightarrow$ K95 & $\mathrm{D} 48 /{ }^{*} \rightarrow \mathrm{K} 134, \mathrm{R} 131$ & $\mathrm{E} 49 \rightarrow \mathrm{K} 41$ \\
\hline $152 \rightarrow \mathrm{K} 95$ & $\begin{array}{l}\mathrm{L} 51 \text { / L51 } \rightarrow \text { E36, D94, T95 } \\
\rightarrow \text { D73, D94 }\end{array}$ & $\mathrm{L} 52 \rightarrow \mathrm{Y} 56, \mathrm{E} 37$ \\
\hline$Y 56 \rightarrow \mathrm{N} 60, \mathrm{E76}$ & Y55 / Y55 $\rightarrow$ H76, G74 & Y56 \\
\hline$R 57 \rightarrow \mathrm{E} 63, \mathrm{~S} 64, \mathrm{D} 74, \mathrm{~N} 60$ & $\begin{array}{l}R 56 / R 56 \\
\rightarrow E 62, A 63, D 73, Y 129 \\
\rightarrow E 62, A 63, A 64, D 73, D 123\end{array}$ & $R 57 \rightarrow \mathrm{D} 63, \mathrm{H} 72$ \\
\hline$N 60 \rightarrow$ S64, E76,Y56,R57 & T59 & $\mathrm{N} 60 \rightarrow \mathrm{N} 60$ \\
\hline $\mathrm{Q} 61 \rightarrow \mathrm{E} 63$ & $\begin{array}{l}\mathrm{Q} 60 / \mathrm{Q} 60 \rightarrow \mathrm{A} 10, \mathrm{Q} 60, \mathrm{E} 62, \\
\mathrm{~A} 63, \mathrm{~A} 64 ; \rightarrow \mathrm{A} 10, \mathrm{Q} 60, \mathrm{~A} 63\end{array}$ & Q61 $\rightarrow$ A11, N60, D64 \\
\hline$E 63 \rightarrow \mathrm{R} 57, \mathrm{~N} 60, \mathrm{Q} 61$ & E62 / E62 $\rightarrow$ R56, Q60, R69 & D63 \\
\hline $\begin{array}{l}S 64 \\
\rightarrow \mathrm{R} 57, \mathrm{~N} 60\end{array}$ & $\begin{array}{l}\text { A63/A63 (backbone) } \\
\rightarrow \text { K13, R18, R56,Q60 }\end{array}$ & $\begin{array}{l}\text { D64 } \\
\rightarrow \mathrm{K} 14, R 19, \mathrm{R} 57, \mathrm{~N} 60, \mathrm{Q} 61\end{array}$ \\
\hline$R 70 \rightarrow \mathrm{E} 76$ & $\mathrm{R} 69 \rightarrow \mathrm{N} 71$ & $\mathrm{R} 70 \rightarrow \mathrm{Q} 61$ \\
\hline$D 74 \rightarrow \mathrm{R} 57$ & $\begin{array}{l}D 73 / D 73 \rightarrow Y 55, R 56, T 59 \\
\rightarrow L 51, \text { A52, Y55, R56 (Zn/Mn) }\end{array}$ & $E 74 \rightarrow S 126, Y 128$ \\
\hline $\begin{array}{l}E 76 \rightarrow \mathrm{G} 51, \mathrm{~T} 54, \mathrm{Y} 56, \mathrm{R} 57, \mathrm{~N} 60, \\
\mathrm{R} 70, \mathrm{~K} 72\end{array}$ & G75 $\rightarrow$ N71 & G76 \\
\hline Q77 & $\begin{array}{l}\mathrm{H76} / \mathrm{H} 76 \rightarrow \mathrm{E} 36, \mathrm{Y} 55, \mathrm{D} 73, \mathrm{H76} \\
\rightarrow \mathrm{E} 36, \mathrm{H} 76, \mathrm{D} 73 \text { (Zn/Mn) }\end{array}$ & K77 \\
\hline $\mathrm{K} 95 \rightarrow \mathrm{K} 44, \mathrm{G} 51, \mathrm{I52}, \mathrm{A} 53, \mathrm{~T} 54$ & D94 / D94 $\rightarrow$ T53 & $\mathrm{D} 95 \rightarrow \mathrm{G} 51$ \\
\hline
\end{tabular}

Table 2: Summary of the interactions between Fur dimers. Colored residues are those found important in windows 16-20: red for major interactions, yellow for medium interaction and blue for minor interactions not described as one of the 15 most important in Figure S7. Residues in Black or italics have large interaction energies when the tetramer is formed (window 0 and neighbors). The five background colors correspond to the structure patches described in the text and localized in Figure 10. Models include residues: Asp 7 to Glu 138 for FtFur; Met 1 to Lys 134 for PaFur, Met 1 to Lys 132 for PaFur -mS3 $\left({ }^{*}\right)$ and Met 1 to Cys 133 for VcFur. 

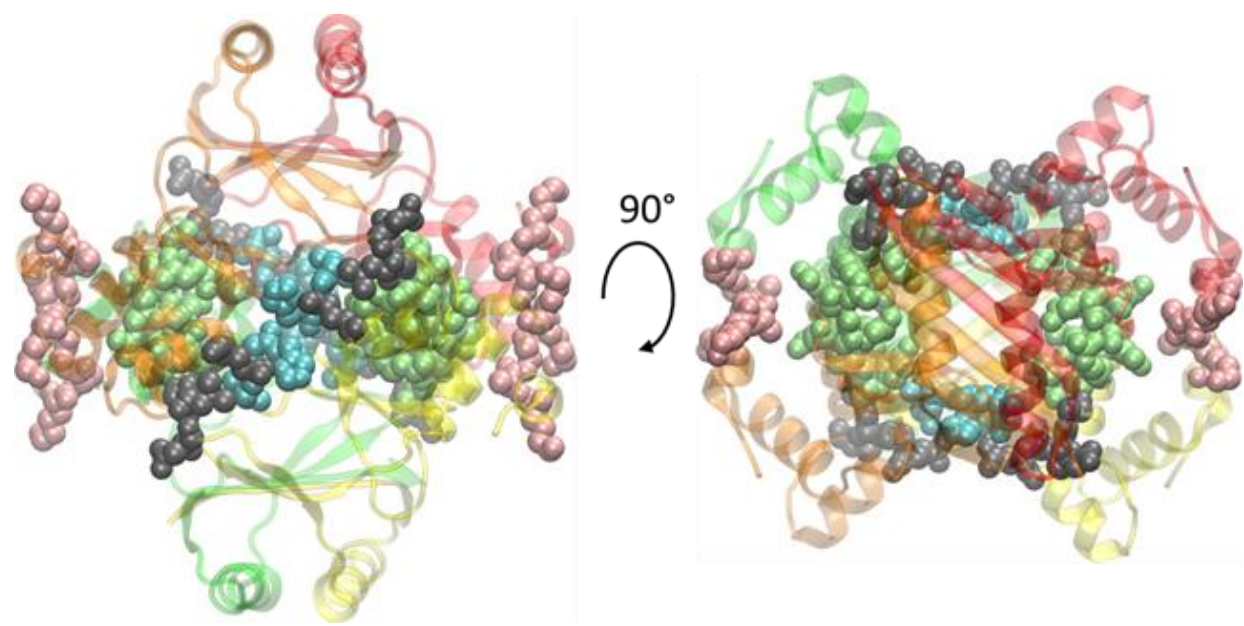

Fig. 10: Patches of interactions between dimers in the tetramers, displayed on the PaFur-mS3 X-ray structure. Patch colors: (1) in pink; (2) in green; (3) in gray;(4) in blue and (5) in cyan. 


\section{Discussion}

PaFur wild type and PaFur H86A-H124A (PaFur-mS3), which are purified as tetramers, do not contain any metal. The S1 zinc site is replaced by an ionic bond between Asp94 and Arg131 in each subunit stabilizing the conformation of a dimer into the tetramer. What stabilizes the apo tetramer cannot be found from the structure alone and the theoretical data together with the comparison with FtFur suggest the importance of the interaction between residues conserved into the tetramers. However, most of these residues are also present in the dimers.

Interestingly, this study highlights the presence of residues like D64 in VcFur, which prevent tetramerization by early interactions during the approach of the two dimers (long distance).This acidic residue is conserved in all the sequences of the known dimers (from biochemical analyses) and absent in the stable tetramers. Moreover as proved by the free energy calculation, the gain in energy between dimer at long distances and the compact tetramer is small in VcFur compared to the true tetramers.

Taken together, the oligomeric division into two groups could be due to several factors, known or not, playing a major role in bacterial metabolism. However, what is certain is that the actual repartition of Fur proteins into dimers and tetramers is probably what suits the best each species in its actual environment. One may argue that, depending on bacterial species, Fur proteins can be involved in slightly different regulatory networks, which can explain the need for different oligomeric states capable of interacting with different partners. In species that produce Fur tetramers, natural selection opted for a tetramer because it should add value that could be the protection of less stable dimers, or the regulation of DNA binding specificity through tetramer/DNA interaction before the dimer/DNA interaction.

In our previous work, the structure in presence of $\mathrm{Mn}^{2+}$ or $\mathrm{Fe}^{2+}$ (its physiological cofactor) showed that FtFur is a pre-activated protein that requires DNA for tetramer dissociation and we deciphered a DNA-driven dissociation mechanisms for these tetrameric proteins preactivated by the metal structure (Perard et al. 2018). In the case of tetrameric Fur, PaFur and FtFur, the presence of DNA alone, triggers the dimerization process on the DNA. This DNA driven dissociation was shown to be non-specific for PaFur and Furbox-specific in the case of FtFur.

In the tetramer built from Pohl's structure, except for some unconventional metal sites found at the interface between two dimers, there is no strong ionic interaction or salt bridge in the structure that would explain the stability of the tetramer, like the two salt bridges between Arg 57 and Glu63 found in the FtFur structure (Perard et al. 2018). This Arg57 is essential for the DNA binding specificity and DNA-driven dissociation process. However, looking at these 
residues in the Mn-PaFur-mS3 tetramer, we found that the four corresponding arginines, Arg 56 , are directly involved in $\mathrm{H}$-bonding of their guanidinium group with the carbonyl of Glu62 and Ala63.

Interestingly the regulons are smaller, (less DNA Fur binding sites), in the strains producing tetramers (hundreds genes regulated by Fur in $\mathrm{Ec} / \mathrm{Vc}$ versus only few in Ft or $\mathrm{Pa}$ ). Knowing that two dimers bind to one Fur box, it may be easier to dissociate a tetramer close to the DNA than to recruit two dimers, which may be far away from one another.

A crown of positive charges in solvent exposed residues of the tetramer could favor a histonelike positioning around the DNA. A specific sequence Fur box would then be able to pull down the two dimers to favor their allosteric binding onto the DNA for regulation of the expression through base-specific interactions with conserved residues such as K14, R57 and Y56.

Interestingly, a new role has been proposed very recently for Helicobacter pylori Fur where oligomers of proteins, such as tetramers, interact with DNA and have a compaction role and a higher level of regulatory function compaction (Roncarati et al. 2016).

Indeed, the question of how these regulatory proteins find, in vivo, their DNA target for regulation is still an open question and a very recent new paradigm has been proposed about this concern, involving the role of protein concentration in the binding-unbinding reaction of protein to DNA (Chen et al. 2018). It would be very interesting to study if such compaction process or concentration dependency (possibly related to oligomer formation) on DNA binding is physiologically relevant in the case of Fur. 


\section{Material and methods}

\section{Phylogeny: tree construction and software}

Amino acid sequences were aligned using Clustal Omega (Sievers et al. 2011). Gblocks (Castresana 2000) was used on the aligned sequences to eliminate poorly aligned positions and divergent regions with a maximum number of contiguous non-conserved positions equal to 8 , minimum length of a block was set to 10 and no gaps were allowed. The generated blocks were used to build the phylogenetic trees in SeaView version 4.7 (Gouy et al. 2010) through the phyML method (Guindon et al. 2010), with 100 replicates, that uses Felsenstein's bootstrapping in phylogenetic tree (Felsenstein 1985, Hillis \& Bull 1993). Protein sequences were obtained from https://www.ncbi.nlm.nih.gov/protein/ with references given in Table S2.

\section{Structure of Fur from P. aeruginosa}

Since the structure of PaFur-WT was already solved in the presence of $\mathrm{Zn}^{2+}$ (PDB ID: 1MZB), trials to get the structural information in the presence of iron, its physiological cofactor, were initiated in anaerobic conditions. After several unsuccessful assays, iron was replaced with $\mathrm{Mn}^{2+}$ to conduct experiments in aerobic condition and at the same time conserve a chemical behavior similar to iron. All crystallization trial were carried out through INEXT PID: 2217 using the crystallization screens of Hampton Research Grid Screens ${ }^{\mathrm{TM}}$ and Qiagen protein crystallization suites at HTXLab high throughput robot screening facility (HTX Lab at EMBLGrenoble). Using the harvester robot, several crystals were tested in order to obtain the first diffraction patterns around $4 \AA$. Following multiple tests, crystals of Mn-PaFur-mS3 were obtained in the crystallization condition of Mn-FtFur, described in Pérard et al., 2018 (Perard et al. 2018), that contained $50 \mathrm{mM}$ MES pH 5.8, 20\% w/v PEG 3350, $200 \mathrm{mM} \mathrm{MgCl}\left(\mathrm{H}_{2} \mathrm{O}\right)_{6}$, $10 \mathrm{mM} \mathrm{MnCl}_{2}$. Before crystal collection, crystals were carefully washed to remove excess free $\mathrm{Mn}^{2+}$ in a solution equivalent to their mother liquor but lacking the metal, before being cryoprotected using a solution obtained by adding $25 \%(\mathrm{v} / \mathrm{v})$ glycerol to the mother liquor. After data analysis, the symmetry space group P6122 was determined in an initial crystallization condition that was later manually optimized to obtain a final diffraction close to $2.34 \AA$.

To validate the presence of metal in our crystals, X-ray fluorescence spectra were realized (data not shown). In Mn-PaFur-mS3 crystals, $\mathrm{Zn}^{2+}$ and $\mathrm{Mn}^{2+}$ signals were detected as expected. However, due to the physical properties of $\mathrm{Zn}^{2+}$ and $\mathrm{Mn}^{2+}$, their discrimination in electron density maps is not obvious. To validate this information, the metal content was measured in the purified proteins by ICP-AES for PaFur-WT and PaFur-mS3. $\mathrm{Zn}^{2+}$ was only 
detected in PaFur-mS3 at 0.8 equivalent per subunit. The absence of $\mathrm{Zn}^{2+}$ in PaFur-WT could be due to the purification protocol involving treatment by EDTA.

The structure of Mn-PaFur-mS3 was solved by molecular replacement using Phenix 1.10.12155 with AutoSol and $95 \%$ of the model was built automatically and manually corrected in Coot before a final refinement cycle in Phenix (Adams et al. 2010). To differentiate the localization of each metal, data sets were collected at $0.99 \AA$ to be able to detect both elements and at $1.29 \AA$ to detect $\mathrm{Mn}^{2+}$ solely (Supplementary Figure S4). With this data collection method, an anomalous map was built where the discrimination between metal elements was performed. $\mathrm{Mn}^{2+}$ atoms were placed in each peak superior to $5 \sigma$ in the anomalous map; this value was arbitrarily chosen at it is data set dependent. Data collection, phasing and refinement statistics generated using XDS/XSCALE (Kabsch 2010) and Phenix (Adams et al. 2010) is shown in Table 1.

\section{Construction of the models}

CHARMM (Brooks et al. 1983) was used to build all initial models of Fur tetramer systems. Its scripting language was useful for building missing coordinates from internal coordinates (IC PARAm, IC BUILd), initial orientation and energy minimization of vacuum systems. The GROMACS program version 5.1.2 (Abraham et al. 2015), with the gromos54a7 united atom force field (Schmid et al. 2011), was used to perform long Molecular Dynamics simulations needed to compute free energy profiles. $\mathrm{Fe}^{2+}$ and $\mathrm{Zn}^{2+}$ were modelled as simple Lennard Jones hard spheres with charge +2 , further referred to as di-cations, with $\mathrm{Zn}^{2+}$ coordinated to charged deprotonated cysteines. The pdb file corresponding to the X-ray structures were prepared for GROMACS with pdb2gmx.

\section{FtFur Tetramer}

The Xray structure of FtFur resolved recently in our laboratory (Asp 7 to Glu 138 PDB ID: $5 \mathrm{NHK}$ ) was used as initial model for the tetramer (Perard et al. 2018). All histidine residues were given type HISA with protonated Nס1 atom. In all 4 protein subunits, cysteins 93, 96, 133 and 136 were deprotonated and given a total charge of -0.75 ( -0.05 for $\mathrm{C} \alpha,-0.15$ for $\mathrm{C} \beta,-0.55$ for $S$ ). This charge can be compared to that of the CYS residue in gromos $54 a 7$ force field, set to -0.5 . A value of -0.75 was adopted instead in this work high enough to stabilize a tetracoordinated Zinc ion providing correct orientation of the cysteins and proper metal environment during further simulations. (Use of a -0.5 charge led to the escape of the metal from its binding site). In addition to zinc, four Fe2+ ions were included in the simulation in FtFur site S2. 


\section{PaFur-Tetramer}

The model of the PaFur tetramer was built from PDB structure 1MZB (Pohl et al. 2003) with the PISA program (Krissinel \& Henrick 2007). The PISA tetramer structure with largest internal free energy $\left(-304.5 \mathrm{kcal}^{\mathrm{mol}}{ }^{-1}\right)$ was selected and 8 zinc cations were constructed with CHARMM in the metal sites described by Pohl (Pohl et al. 2003). The model includes the full protein from residues Met 1 to Lys 134 .

\section{PaFur-mS3-Tetramer}

A model of the PaFur tetramer without S3 metal site by mutating Histidine 86 and Histidine 124 into Alanine was obtained from the crystal structure recently resolved in our laboratory in the presence of zinc. Again, a PISA tetramer was selected with $8 \mathrm{Zn}^{2+}$ cations chosen from the 21 initial metal atoms in the PISA model. The zinc cations fill in the S2 metal site (H32, E80, H89 and E100) and the incomplete S3 site reduced to D88 and E107. The model includes residues Met 1 to Lys 132 (the last 2 residues being unresolved in the X-ray structure).

\section{VcFur tetramer}

Although it does not form in the case of VcFur, a tetramer was built from the structure of PaFur, as a negative control to this study. VcFur contains 150 amino acids from Met1 to Lys 150 but the crystal structure was resolved for residues Asp 3 to Cys 133, only. A VcFur dimer model (chains $A, B$ ) extending from residues Met 1 to Cys 133 was built and energy minimized with CHARMM from PDB structure 2W57 (Sheikh \& Taylor 2009). Coordinates for Met 1 and Ser 2 were constructed from internal coordinates with CHARMM. The structure of the tetramer was built starting from a sequence alignment between VcFur and PaFur. The coordinates of the PaFur tetramer built with PISA and described previously was used as template for the building of a potential VcFur tetramer. The coordinates of all backbone atoms $(\mathrm{N}, \mathrm{CA}, \mathrm{C})$ of residues of the VcFur dimer and of PaFur (chains A, B) showing a sequence identity were superimposed with a home-made program. 432 atoms corresponding to 144 sequence identities were superimposed with a rms deviation of $3.75 \AA$. Then the PaFur dimer $(A, B)$ was superimposed with the other PaFur dimer (Chains $C, D)(r m s d=0)$ to yield a superposition matrix which was applied on VcFur $(A, B)$ to yield a second VcFur dimer (chains $C, D$ ) in the new tetramer. 
The complexes studied are shown in Table 3.

\begin{tabular}{|l|l|l|l|l|l|l|}
\hline System & $\begin{array}{l}\text { Box size } \\
\mathrm{nm}^{3}\end{array}$ & $\begin{array}{l}\text { Num. } \\
\text { Metal } \\
\mathrm{M}^{2+}\end{array}$ & $\begin{array}{l}\text { Num. } \\
\mathrm{Na}^{+}\end{array}$ & $\begin{array}{l}\text { Num. } \\
\mathrm{Cl}^{-}\end{array}$ & $\begin{array}{l}\text { Num. } \\
\text { atoms }\end{array}$ & $\begin{array}{l}\text { PDB } \\
\text { ID }\end{array}$ \\
\hline PaFur-Tetramer & $10.2^{\star} 8.7^{*} 6.9$ & 8 & 44 & 36 & 57337 & $1 \mathrm{MZB}$ \\
\hline $\begin{array}{l}\text { PaFur-mS3 } \\
\text { Tetramer }\end{array}$ & $10.4^{\star} 8.6^{*} 6.8$ & 4 & 53 & 37 & 58232 & $\begin{array}{l}\text { this } \\
\text { work }\end{array}$ \\
\hline FtFur-Tetramer & $10.5^{\star} 8.0^{*} 6.5$ & 8 & 37 & 33 & 52114 & $5 \mathrm{NHK}$ \\
\hline $\begin{array}{l}\text { VcFur-Tetramer } \\
\text { (model) }\end{array}$ & $10.4^{\star} 8.9^{\star} 7.3$ & 8 & 55 & 41 & 64287 & - \\
\hline
\end{tabular}

Table 3: Size and ion composition of the systems studied. Metal dications are represented as charged van der Waals spheres that do not discriminate between different atoms in our simple models of the metal binding sites. Number of counterions and total number of atoms are indicated.

The four above vacuum systems were immersed in parallelepipedic SPC water boxes modelled with periodic boundary conditions after the addition of $\mathrm{Na}^{+}$and $\mathrm{Cl}^{-}$counterions to ensure neutrality and a total ionic force of $0.1 \mathrm{~mol}^{-\mathrm{L}^{-1}}$. The solvated systems were energy minimized and equilibrated under NPT (constant Number of particles, Pressure and Temperature) conditions at $310 \mathrm{~K}$ and $1 \mathrm{~atm}$ and a time step of $2 \mathrm{fs}$ was used.

\section{Calculation of interaction energies}

The gromos force field includes four non bonded energy terms, only two of them (Coul-SR and LJ-SR) were used in our calculations. To make sure that we could neglect the coulomb reciprocal and dispersion correction terms in our analysis, the four energy terms were calculated as a function of time for the whole system in the case of the starting conformations of the FtFur tetramer complex. We found that the coulomb reciprocal and dispersion correction terms contribute only $0.3 \%$ to the total potential energy meaning that they could effectively be neglected in comparison to the electrostatic and LJ potential terms.

\section{Computation of free energy profiles}

Free energy profiles for the extraction (by translation along a fixed direction) of one Fur dimer from the tetramer were computed. The simulations include a 'moving' subsystem (Fur dimer, chains $A$ and $B$ ) and a 'fixed' subsystem (Fur dimer, chains $C$ and D) as shown in 
Supplementary Figure 7 of Perard et al (Perard et al. 2018) in the case of the FtFur tetramer simulation.

The free energy profiles were built using the 'umbrella sampling' technique (Crouzy et al. 1994), and result from the overlapping of 26 computation windows that cover the reaction path of our systems. They are generated by translating the moving subsystem from the initial conformation along the $X$ axis, in order to create snapshots of conformations during the dissociation. There is one computation window for each translation distance. The meticulous translation protocol is shown in Suppl. Fig. 6 of Perard et al(Perard et al. 2018). Each window consisted of 100 ps NPT equilibration and 10 to 15 ns NPT production simulations. Position restraints on the 'fixed' subsystem and distance restraints on the whole protein, in the form of NOE-type restraints (Nuclear Overhauser Effect) between $\mathrm{H}$-bonded $\mathrm{H}$ and $\mathrm{O}$ atoms to maintain its secondary structure, were applied. The 'moving' subsystem was subject to two harmonic biasing forces along the $X$ direction only ('umbrella potential') applied between the centers of mass of the 2 Fur dimer subunits and the center of mass of the 'fixed' subsystem. In both equilibration and production umbrella sampling MD simulations, the system was simulated under NPT conditions. Temperature was fixed at $310 \mathrm{~K}$ with Temperature coupling using a Nose-Hoover extended ensemble with $\mathrm{T}_{\mathrm{T}}=0.5 \mathrm{ps}$ (Hoover 1985, Nosé 1984), Pressure was controlled at 1 atm with extended-ensemble Parrinello-Rahman isotropic pressure coupling with $\mathrm{TP}_{\mathrm{P}}=1 \mathrm{ps}$ and compressibility $=4.5 \times 10^{-5} \mathrm{bar}^{-1}$ (Parrinello \& Rahman 1981). After the dynamics runs, positions and forces were collected from the trajectories and the umbrella sampling harmonic potential was unbiased using the Wham algorithm(Kumar et al. 1992) implemented in the g-wham program (Hub et al. 2010) to yield the free energy profiles. Calculations were carried on until good convergence and reliable error estimation could be obtained. After 60 ns simulation time per window, the binding free energies were estimated. 


\section{Bibliography}

Abraham, M. J., Murtola, T., Schulz, R., Pall, S., Smith, J. C., Hess, B., and Lindahl, E. (2015) GROMACS: High performance molecular simulations through multi-level parallelism from laptops to supercomputers, Journal of chemical theory and computation 11, 19-25.

Adams, P. D., Afonine, P. V., Bunkoczi, G., Chen, V. B., Davis, I. W., Echols, N., Headd, J. J., Hung, L. W., Kapral, G. J., Grosse-Kunstleve, R. W., McCoy, A. J., Moriarty, N. W., Oeffner, R., Read, R. J., Richardson, D. C., Richardson, J. S., Terwilliger, T. C., and Zwart, P. H. (2010) PHENIX: a comprehensive Python-based system for macromolecular structure solution, Acta crystallographica. Section D, Biological crystallography 66, 213-221.

Agriesti, F., Roncarati, D., Musiani, F., Del Campo, C., lurlaro, M., Sparla, F., Ciurli, S., Danielli, A., and Scarlato, V. (2014) FeON-FeOFF: the Helicobacter pylori Fur regulator commutates ironresponsive transcription by discriminative readout of opposed DNA grooves, Nucleic acids research 42, 3138-3151.

Beauchene, N. A., Mettert, E. L., Moore, L. J., Keles, S., Willey, E. R., and Kiley, P. J. (2017) O2 availability impacts iron homeostasis in Escherichia coli, Proceedings of the National Academy of Sciences of the United States of America 114, 12261-12266.

Brooks, B. R., Bruccoleri, R. E., Olafson, B. D., States, D. J., Swaminathan, S., and Karplus, M. (1983) CHARMM: a program for macromolecular energy, minimization, and dynamics calculations., $J$. Comp. Chem. 4, 187-217.

Castresana, J. (2000) Selection of conserved blocks from multiple alignments for their use in phylogenetic analysis, Molecular biology and evolution 17, 540-552.

Chen, T.-Y., Cheng, Y.-S., Huang, P.-S., and Chen, P. (2018) Facilitated Unbinding via MultivalencyEnabled Ternary Complexes: New Paradigm for Protein-DNA Interactions, Acc. Chem. Res. 51, 860-868.

Crouzy, S., Woolf, T. B., and Roux, B. (1994) A molecular dynamics study of gating in dioxolane-linked gramicidin A channels, Biophysical journal 67, 1370-1386.

D'Autreaux, B., Pecqueur, L., Gonzalez de Peredo, A., Diederix, R. E., Caux-Thang, C., Tabet, L., Bersch, B., Forest, E., and Michaud-Soret, I. (2007) Reversible redox- and zinc-dependent dimerization of the Escherichia coli fur protein, Biochemistry 46, 1329-1342.

Delany, I., Spohn, G., Pacheco, A. B., leva, R., Alaimo, C., Rappuoli, R., and Scarlato, V. (2002) Autoregulation of Helicobacter pylori Fur revealed by functional analysis of the iron-binding site, Mol Microbiol 46, 1107-1122.

Deng, Z., Wang, Q., Liu, Z., Zhang, M., Machado, A. C., Chiu, T. P., Feng, C., Zhang, Q., Yu, L., Qi, L., Zheng, J., Wang, X., Huo, X., Qi, X., Li, X., Wu, W., Rohs, R., Li, Y., and Chen, Z. (2015) Mechanistic insights into metal ion activation and operator recognition by the ferric uptake regulator, Nature communications 6, 7642 .

Dian, C., Vitale, S., Leonard, G. A., Bahlawane, C., Fauquant, C., Leduc, D., Muller, C., de Reuse, H., Michaud-Soret, I., and Terradot, L. (2011) The structure of the Helicobacter pylori ferric uptake regulator Fur reveals three functional metal binding sites, Mol Microbiol 79, 1260-1275.

Efron, B. (1979) Bootstrap Methods: Another Look at the Jackknife, Ann. Statist. 7, 1-26.

Felsenstein, J. (1985) Confidence Limits on Phylogenies: An Approach Using the Bootstrap, Evolution; international journal of organic evolution 39, 783-791.

Gouy, M., Guindon, S., and Gascuel, O. (2010) SeaView version 4: A multiplatform graphical user interface for sequence alignment and phylogenetic tree building, Molecular biology and evolution 27, 221-224.

Guindon, S., Dufayard, J. F., Lefort, V., Anisimova, M., Hordijk, W., and Gascuel, O. (2010) New algorithms and methods to estimate maximum-likelihood phylogenies: assessing the performance of PhyML 3.0, Systematic biology 59, 307-321.

Hillis, D. M., and Bull, J. J. (1993) An Empirical Test of Bootstrapping as a Method for Assessing Confidence in Phylogenetic Analysis, Systematic biology 42, 182-192.

Hoover, G. (1985) Canonical dynamics: Equilibrium phase-space distributions, Phys. Rev. A31, 1695. 
Hub, J. S., de Groot, B. L., and van der Spoel, D. (2010) GROMACS g_wham A Free Weighted Histogram Analysis Implementation Including Robust Error and Autocorrelation Estimates, J. Chem. Theory Comput. 6, 3713.

Kabsch, W. (2010) XDS, Acta crystallographica. Section D, Biological crystallography 66, 125-132.

Krissinel, E., and Henrick, K. (2007) Inference of macromolecular assemblies from crystalline state, Journal of molecular biology 372, 774-797.

Kumar, S., Rosenberg, J. M., Bouzida, D., Swendsen, R. H., and Kollman, P. A. (1992) The Weighted Histogram Analysis Method for Free-Energy Calculations on Biomolecules. I., The Method J. Comput. Chem. 13, 1011.1021.

Le Cam, E., Frechon, D., Barray, M., Fourcade, A., and Delain, E. (1994) Observation of binding and polymerization of Fur repressor onto operator-containing DNA with electron and atomic force microscopes, Proceedings of the National Academy of Sciences of the United States of America 91, 11816-11820.

Nosé, S. (1984) A unified formulation of the constant temperature molecular dynamics methods, $J$. Che. Phys. 81, 511.

Parrinello, M., and Rahman, A. (1981) Polymorphic transitions in single crystals: A new molecular dynamics method, J. Appl. Phys 52, 7182-7190.

Perard, J., Coves, J., Castellan, M., Solard, C., Savard, M., Miras, R., Galop, S., Signor, L., Crouzy, S., Michaud-Soret, I., and de Rosny, E. (2016) Quaternary Structure of Fur Proteins, a New Subfamily of Tetrameric Proteins, Biochemistry 55, 1503-1515.

Perard, J., Nader, S., Levert, M., Arnaud, L., Carpentier, P., Siebert, C., Blanquet, F., Cavazza, C., Renesto, P., Schneider, D., Maurin, M., Coves, J., Crouzy, S., and Michaud-Soret, I. (2018) Structural and functional studies of the metalloregulator Fur identify a promoter-binding mechanism and its role in Francisella tularensis virulence, Communications biology 1, 93.

Pi, H., and Helmann, J. D. (2017) Sequential induction of Fur-regulated genes in response to iron limitation in Bacillus subtilis, Proceedings of the National Academy of Sciences of the United States of America 114, 12785-12790.

Pohl, E., Haller, J. C., Mijovilovich, A., Meyer-Klaucke, W., Garman, E., and Vasil, M. L. (2003) Architecture of a protein central to iron homeostasis: crystal structure and spectroscopic analysis of the ferric uptake regulator, Mol Microbiol 47, 903-915.

Roncarati, D., Pelliciari, S., Doniselli, N., Maggi, S., Vannini, A., Valzania, L., Mazzei, L., Zambelli, B., Rivetti, C., and Danielli, A. (2016) Metal-responsive promoter DNA compaction by the ferric uptake regulator, Nature communications 7, 12593.

Sarvan S., Butcher J., Stintzi A., Couture J.F. (2018) Variation on a theme: investigating the structural repertoires used by ferric uptake regulators to control gene expression, Biometals 31(5) 681704.

Schmid, N., Eichenberger, A. P., Choutko, A., Riniker, S., Winger, M., Mark, A. E., and van Gunsteren, W. F. (2011) Definition and testing of the GROMOS force-field versions $54 A 7$ and 54B7, European biophysics journal : EBJ 40, 843-856.

Seo, S. W., Kim, D., Latif, H., O'Brien, E. J., Szubin, R., and Palsson, B. O. (2014) Deciphering Fur transcriptional regulatory network highlights its complex role beyond iron metabolism in Escherichia coli, Nature communications 5, 4910.

Sheikh, M. A., and Taylor, G. L. (2009) Crystal structure of the Vibrio cholerae ferric uptake regulator (Fur) reveals insights into metal co-ordination, Mol Microbiol 72, 1208-1220.

Sievers, F., Wilm, A., Dineen, D., Gibson, T. J., Karplus, K., Li, W., Lopez, R., McWilliam, H., Remmert, M., Soding, J., Thompson, J. D., and Higgins, D. G. (2011) Fast, scalable generation of highquality protein multiple sequence alignments using Clustal Omega, Molecular systems biology 7,539 . 